\title{
Use of advance directives by South Australians: results from the Health Omnibus Survey Spring 2012
}

\section{Sandra L Bradley \\ BSC, BN, MSC \\ PhD Candidate, Palliative and \\ Supportive Services

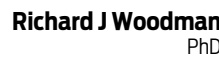 \\ Professor, Flinders Centre \\ for Epidemiology and Biostatistics \\ Jennifer J Tieman BSc(Hons), MBA, PhD CareSearch Director, Palliative and Supportive Services \\ Paddy A Phillips MBBS, DPhil, FRACP Professor of Medicine \\ Flinders University, Adelaide, SA \\ sandra.bradley@ \\ flinders.edu.au}

MJA 2014; 201: 467-469 doi: 10.5694/mjal3.00175 dvance directives (ADs) have been championed as a remedy for providing evidence of the medical, financial, health care and lifestyle options a person would choose at a time when they may lack capacity for decision making. ${ }^{1}$ A review of the literature in this area found that these instruments can be useful, but are not widely used due to a variety of factors which inhibit their effectiveness. ${ }^{1,2}$ In Australia, barriers to effective completion of ADs include poor understanding and application of the different AD forms. ${ }^{2}$ There is a lack of actual published data on completion rates of specific types of ADs used in Australia. ${ }^{2}$ As primary care involves assessing individuals at increased risk of chronic diseases and hospital care, low rates of completion of ADs in particular age or other demographic groups may suggest the potential for AD completion initiatives led by general practitioners. This would, in turn, lead to better outcomes from a primary health care, patient management and family carer perspective.

The aim of this study was to determine the proportion of South Australians who have completed the medical, financial, health care or lifestyle ADs in South Australia and/or a will (an instrument often believed to be an $\mathrm{AD}^{2}$ ). Demographic variables associated with completion or noncompletion of these instruments were also investigated.

\section{Methods}

Data were collected through the South Australian Health Omnibus Survey (HOS) from 5200 households selected for interview between 4 September and 12 December (spring) 2012. The HOS included a non-replacement sample of people aged 15 years or older. Participants in the spring 2012 survey had an age range of 15 to 97 years. Complete methods for the HOS have been described in detail elsewhere. ${ }^{3}$ In brief, a random

\section{Abstrac}

Objective: To determine the prevalence of completion of advance directives (ADs) and wills by South Australians aged 15 years and over.

Design, setting and participants: Statewide population-based survey of a single member (aged 15 years and over) of 3055 South Australian households between 4 September and 12 December 2012.

Main outcome measures: Prevalence and sociodemographic determinants of completion of the four recognised legal ADs in South Australia (enduring power of attorney [EPA] for finance, enduring power of guardianship [EPG] for health care/lifestyle, medical power of attorney [MPA] for medical treatment and anticipatory direction for end-of-life care) and wills.

Results: Nearly half the 3055 survey participants had not completed any AD document or will. Financial documents were more likely to be completed than health care documents. In multivariate analysis, the odds of not having completed any AD was higher among those aged 15-24 years compared with those aged over 65 years (odds ratio [OR], 55.3; 95\% Cl, 31.3-97.7) and 25-44 years (OR, 24.9; $95 \% \mathrm{CI}, 17.3-36.1)$. Similarly, the odds were higher for those born in another country (OR, 2.0; $95 \% \mathrm{Cl}, 1.6-2.4$ ); those never married (OR, 3.1; 95\% $\mathrm{Cl}, 2.3-4.2)$ or in de facto relationships (OR, 1.8; 85\% Cl, 1.3-2.4) or separated/ divorced (OR, 2.8; 95\% Cl, 2.1-3.8) compared with those married; those who left school in Year 12 or before (OR, 1.5; 95\% Cl, 1.1-1.9) or with a bachelor degree (OR, 1.5; 95\% Cl, 1.1-2.0) compared with those who had completed a trade/ apprenticeship, certificate/diploma; and those in blue collar occupations (OR 1.6; $95 \% \mathrm{Cl}, 1.3-2.2$ ) or not employed (OR, 2.2; 95\% Cl, 1.4-3.6) versus professionals. The odds of not having completed any AD were lower for those living in rural or regional areas (OR, $0.8,95 \% \mathrm{Cl}, 0.6-1.0$ ) compared with the metropolitan area, and for those in the highest income bracket earning more than $\$ 80000$ (OR, 0.6; $95 \% \mathrm{Cl}, 0.5-0.8$ ) compared with those in the middle bracket earning \$40000-\$80000.

Conclusion: Completion rates of ADs among South Australians remain low, with financial instruments more likely to be completed than health care and lifestyle instruments. The odds of not completing ADs were associated with age and socioeconomic characteristics. General practitioners are in a good position to target advance care planning towards relevant patient groups, which would likely improve rates of decision making in future health care.

stratified sampling technique was used, defined by a random starting point with 10 households sampled per collection district using a fixedskip interval. Data were stratified by sex (male/female), age (18-24, 25-34, $35-44,45-54,55-64$ and $\geqslant 65$ years) and area of residence (metropolitan/ rural/regional). In non-metropolitan locations, samples were self-weighting. A cluster size of 10 households was also used for each of the 130 collection districts. Data were weighted by the inverse of the individual's probability of selection as well as by the response rate in metropolitan and country regions, then reweighted to benchmarks derived from the 2011 estimated resident population based on the 2011 Census, providing a demographic description of the population by age and sex.

All variables were weighted to better align each individual case with the distributions of age, sex and metropolitan or rural/regional location for the total population.

In this study, we report the findings from one survey question relating to the type of AD completed by respondents. Four legal ADs are recognised in SA - enduring power of attorney (EPA), enduring power of guardianship (EPG), medical power of attorney (MPA) and anticipatory direction.

To clarify the specific documents in question, an introductory statement 


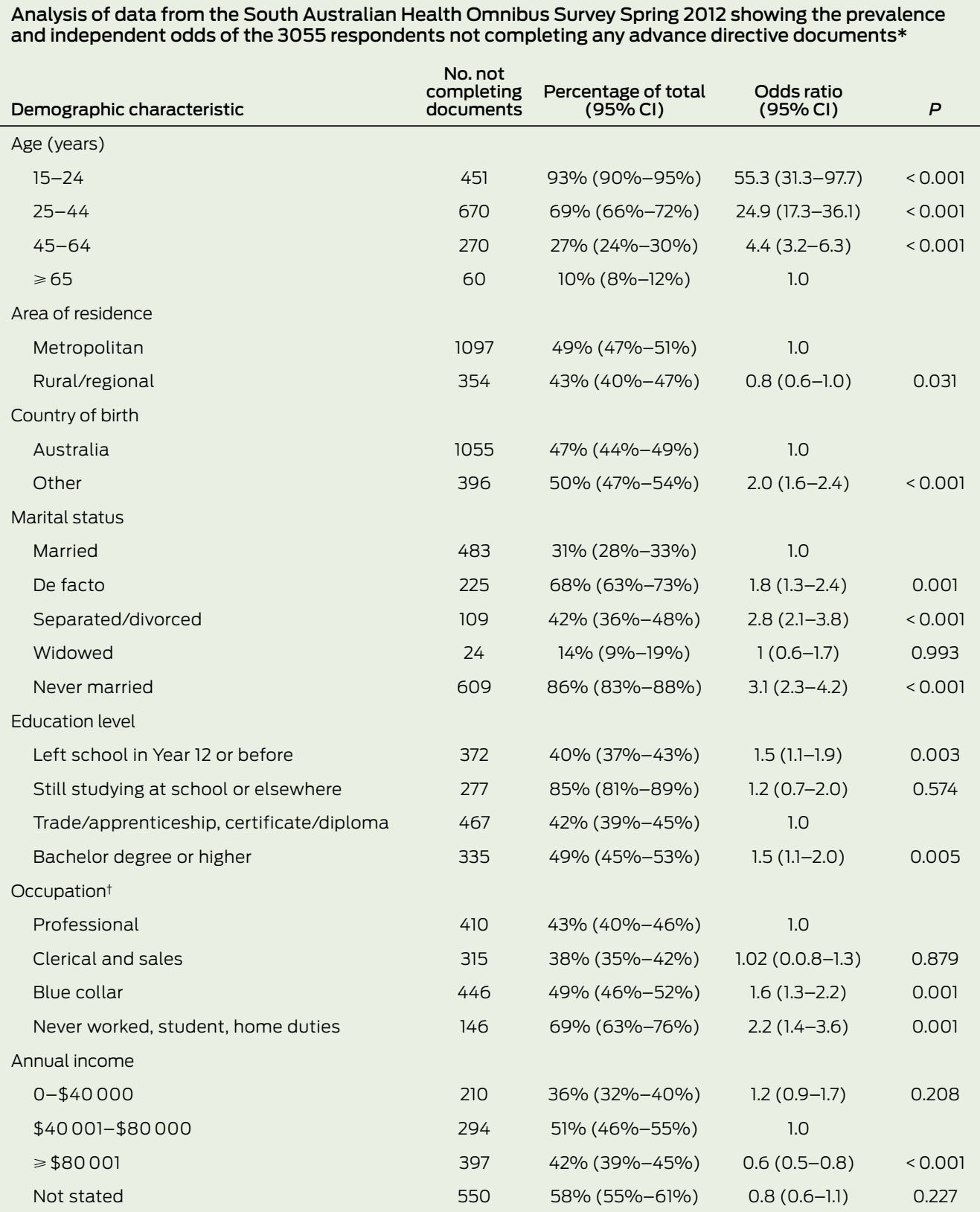

* Multivariate binary logistic regression analysis; numbers and percentages may not add to totals or $100 \%$ as data are weighted and rounded to whole decimals. †Australian and New Zealand Standard Classification of Occupations codes. ${ }^{4}$

about ADs in general and a brief definition of each $\mathrm{AD}$ was provided to participants at the interview stage (Appendix 1; online at mja.com.au). We used the term "living will" instead of anticipatory direction in this introductory statement as the living will has had more consistent use in the literature and by the public. ${ }^{1,2}$ However, for the purposes of this report, we use anticipatory direction to mean any type of living will document rather than it being limited to medical decisions about end of life.
The study was approved by the Flinders University Social and Behavioural Research Ethics Committee (Project No. 5748).

\section{Statistical analysis}

Data were analysed using SPSS version 19 (IBM SPSS Statistics). All analyses were performed using weighted data, and only weighted results are reported unless otherwise specified. Univariate analysis of individual AD document completion with demographic information was performed using $\chi^{2}$ tests of association. A multivariate binary logistic regression was used to assess the independent associations between non-completion of any of the documents and demographic variables. All demographic variables were included in the multivariate model regardless of their statistical significance. Associations for which a two-tailed $P$ value of $<0.05$ were obtained were considered statistically significant.

\section{Results}

\section{Frequency of document completion}

From the 5063 households contacted, 3055 interviews were completed, representing a $60.3 \%$ response rate and a $64.4 \%$ participation rate (Appendix 2; online at mja.com.au). Wills were the most commonly reported completed instruments (48\%), even though they are not ADs. For the recognised legal ADs in SA, more respondents reported having completed the EPA $(22 \%)$ than any of the health care-related documents - EPG (13\%), MPA $(11 \%)$, anticipatory direction $(12 \%)$ (Appendix 3; online at mja.com.au).

\section{Individual document completion}

Appendix 3 shows that there was an inverse association between rates of completion of individual documents and age across all documents, with those aged 65 years and older showing a much higher completion rate than those in the youngest age category of 15-24 years. People who were married or widowed were more likely to have completed the financial documents - EPA (29\% married and $54 \%$ widowed) and a will (65\% married and $81 \%$ widowed) - compared with those who had never married, or who had separated or divorced (EPA, $4 \%$ never married and $10 \%$ separated/ divorced; will, $11 \%$ never married and $27 \%$ separated/divorced; $P<0.001$ for each). There was no difference between the sexes in completion rates of documents, or between metropolitan and rural/regional respondents, except for the EPA and will where the frequency of rural/regional respondent completion rates was slightly higher than that for metropolitan respondents (EPA, 26\% rural/regional v $20 \%$ metropolitan; 
$P=0.002$, and will, $53 \%$ rural v $47 \%$ metropolitan; $P=0.004)$. Those still studying at school or elsewhere had the lowest percentage of completion of documents compared with those with other education levels. Those in the "never worked, student, home duties" category had the lowest percentage compared with those earning an income (results not shown).

\section{Non-completion of any documents}

The Box shows the results of the multivariate binary logistic regression for the reported non-completion of any documents. Non-completion rates for any of the four types of $\mathrm{AD}$ documents were significantly and independently associated with age $(P<0.001)$, area of residence $(P=0.031)$, country of birth $(P<0.001)$, marital status $(P<0.001)$, education level $(P=0.003)$, occupation $(P=0.001)$ and annual income $(P<0.001)$.

\section{Discussion}

Our findings show for the first time that, although completion rates remain highest in the 65 years and older age group, there is still a significant proportion of older people not completing health care ADs. Older people may not be aware that these documents exist, may not accept their role and value, or may choose not to complete them. Some older people may assume or prefer that health professionals will initiate discussions around these documents at the appropriate time. ${ }^{5}$

It is interesting and encouraging that younger people (aged 45-64 years) seem to be completing ADs. GPs and other health care professionals are likely to have increasing and ongoing patient encounters with generational groups like the baby boomers (born 1946-1965) as they age. Providing an opportunity to discuss ADs with those aged 45 years and over can facilitate completion of ADs at a time when patients are in relatively good health, as well as build an understanding of factors that may influence health care decision making for them in the future.

While anyone aged over 18 years can complete an AD in SA, those aged 18 to 45 years are far less likely to complete these documents. Targeted health promotion in this group similar to the recent organ donation campaign could increase awareness and promote the benefits of completed ADs in the event of a health care crisis in the future.

The documents most often reported as completed by the general public were a will and, of the four formal ADs in SA, the EPA, a financial instrument, rather than the health care instruments EPG, MPA and anticipatory direction or living will. The relatively high completion rate of EPAs and wills indicates that taking care of our financial assets is an accepted responsibility for many in the community. This could provide a useful context for encouraging people to complete other life-planning documents when GPs are made aware of life-plan changes for a patient (eg, admission to residential aged care, retirement or travelling overseas or interstate). Being open to discussing ADs at this time may provide future benefits to patients, health care practitioners, and families if a future health care crisis occurs.

Given that rural and regional area of residence and non-married or nonworking status were each associated with reduced odds of completing most ADs, GPs working in the country or in areas with a high proportion of non-married and non-working individuals might find it especially useful to promote the role of ADs and the value of identifying a trusted substitute decisionmaker to help with future decisions.

While our study has shown that rates of completion of ADs, especially health care and lifestyle ADs, continue to be low, the use of these documents by younger age groups is encouraging and indicates that broadening GP discussion of ADs to include younger age groups might be important in meeting patient goals of future care. Our findings also indicate that those in lower socioeconomic groups may be vulnerable to having their personal autonomy in health care decision making thwarted unless they are given targeted direction on ADs. Detail about the sociodemographic characteristics of those most likely not to have completed ADs provides a useful framework that could help GPs provide these groups with targeted health promotion using relevant community health services.

Acknowledgements: This study is part of a $\mathrm{PhD}$ thesis sponsored by the CareSearch project. CareSearch contributed to the financial costs associated with the HOS data collection. CareSearch is funded by the Australian Government Department of Health. We thank Dr Pawel Skuza (Flinders University) for providing assistance in using SPSS and developing the HOS questions used for this study.

Competing interests: No relevant disclosures.

Received 3 Dec 2013, accepted 15 Apr 2014.

1 Wilkinson A, Wenger N, Shugarman L; Rand Corporation. Literature review on advance directives. Washington, DC: United States Department of Health and Human Services, 2007. http://aspe.hhs.gov/daltcp/ reports/2007/advdirlr.htm (accessed Mar 2014).

2 Clinical Technical and Ethical Principal Committee, Australian Health Ministers' Advisory Council. A national framework for advance care directives. Adelaide: AHMAC, 2011. http://www.ahmac.gov.au/cms_documents/ AdvanceCareDirectives2011.pdf (accessed Mar 2014).

3 Wilson D, Wakefield MA, Taylor A. The South Australian Health Omnibus Survey. Health Promot J Austr 1992; 2: 47-49.

4 Australian Government Department of Immigration and Border Protection. ANZSCO Codes and Occupations. Australian and New Zealand Standard Classification of Occupations (ANZSCO). https://www.immi.gov.au/ employers/anzsco/a (accessed Sep 2014).

5 Robinson C, Kolesar S, Boyko M, et al. Awareness of do-not-resuscitate orders: what do patients know and want? Can Fam Physician 2012; 58: e229-e233. 